\title{
A set of four simple performance measures reflecting adherence to guidelines predicts hospitalization: a claims-based cohort study of patients with diabetes
}

\author{
This article was published in the following Dove Press journal: \\ Patient Preference and Adherence \\ I March 2016 \\ Number of times this article has been viewed
}

\section{Carola A Huber' \\ Michael Brändle ${ }^{2}$ \\ Roland Rapold' \\ Oliver Reich' \\ Thomas Rosemann ${ }^{3}$}

'Department of Health Sciences, Helsana Group, Zürich, ${ }^{2}$ Division of Endocrinology and Diabetes, Department of Internal Medicine, Kantonsspital St Gallen, St Gallen, ${ }^{3}$ Institute of Primary Care, University of Zürich, University Hospital Zürich, Zürich, Switzerland
Correspondence: Carola A Huber Department of Health Sciences, Helsana Group, PO Box, 808I Zürich, Switzerland

Tel +4l 43340634 |

Fax +4I 433400434

Email carola.huber@helsana.ch
Background: The link between guideline adherence and outcomes is a highly demanded issue in diabetes care. We aimed to assess the adherence to guidelines and its impact on hospitalization using a simple set of performance measures among patients with diabetes.

Methods: We performed a retrospective cohort study, using health care claims data for adult patients with treated diabetes (2011-2013). Patients were categorized into three drug treatment groups (with oral antidiabetic agents [OAs] only, in combination with insulin, and insulin only). Performance measures were based on international established guidelines for diabetes care. Multivariate logistic regression models predicted the probability of hospitalization (2013) by adherence level (2011) among all treatment groups.

Results: A total of 40,285 patients with diabetes were enrolled in 2011. Guideline adherence was quite low: about $70 \%$ of all patients received a biannual hemoglobin $\mathrm{A}_{1 \mathrm{c}}$ measurement and $19.8 \%$ had undergone an annual low-density lipoprotein cholesterol test. Only $4.8 \%$ were exposed to full adherence including all performance measures (OAs: $3.7 \%$; insulin: $7.7 \%$; and in combination: $7.2 \%$ ). Increased guideline adherence was associated with decreased probability of hospitalization. This effect was strongest in patients using OAs and insulin in combination.

Conclusion: Our study showed that measures to reflect physicians' guideline adherence in diabetes care can easily be calculated based on already available datasets. Furthermore, these measures are clearly linked with the probability of hospitalization suggesting that a better guideline adherence by physicians could help to prevent a large number of hospitalizations.

Keywords: diabetes, adherence, guidelines, hospitalization, antidiabetic agents, insulin

\section{Introduction}

The link between guideline adherence, in terms of performed process measures, and clinical outcomes is a highly demanded issue in diabetes care. Diabetes is one of the leading health problems in the 21 st century and requires continuous medical care, patients' self-management, and risk reduction strategies. ${ }^{1-3}$ Several multidisciplinary expert committees, including the American Diabetes Associations, the European Society of Cardiology, and the European Association for the Study of Diabetes, report and adapt annually clinical guidelines to improve diabetes outcomes. ${ }^{3,4}$ In view of the big discussion on how to measure guideline adherence, evidence suggests that intermediate outcomes such as hemoglobin $\mathrm{A}_{1 \mathrm{c}}\left(\mathrm{HbA}_{1 \mathrm{c}}\right)$ can be improved and complications of diabetes can be reduced by controlling for even a simple set of performance measures such as $\mathrm{HbA}_{1 \mathrm{c}}$, blood pressure, and low-density lipoprotein (LDL) cholesterol. ${ }^{5-8}$ 
However, only few data exist on the adherence to guidelines and its effect on further important clinical outcomes. While studies have shown that the adherence to guidelines has a positive impact on all-cause mortality, the effect on the risk of hospitalization has not been well studied. ${ }^{9,10}$ One study found an association between the adherence to screening guidelines and the hospitalization for complications with diabetes using an outdated (1994-1999) and limited sample of Medicare enrollees aged over 65 years. ${ }^{11}$ Another study with diabetes patients living in a city of north-west Italy and aged 36-80 years showed that adherence was associated with reduced hospitalizations. ${ }^{12}$ Thus, it is not clear how the performance of the most important process measures in diabetes care affects the risk of hospitalization in a large population of elderly and nonelderly patients with diabetes. Furthermore, we assume that it is crucial to differentiate the patients according to drug treatment, since physicians' awareness and therefore guideline adherence increased with therapy intensity. Therefore, the purpose of this study was to examine the adherence to established diabetes guidelines using a set of simple performance measures at individual patient level, and its impact on subsequent hospitalization by differentiating patients by drug treatment. To do so, we used health care claims data for retrospective cohort reflecting a large population with diabetes.

\section{Materials and methods}

\section{Study design and study population}

This study was based on health care claims data from a large health insurance group in Switzerland (Helsana) covering about 1.2 million residents with mandatory health insurance. Claims files contained information on the enrollees' age, sex, health insurance plan, date of hospitalization, and prescribed drugs including the ingredients as defined by the Anatomical Therapeutic Chemical (ATC) code from the World Health Organization. ${ }^{13}$ The health insurance plan was characterized by the choice of a managed care model and the chosen deductible class. As managed care models, we defined health plans with capitation, family doctor models, or telemedicine models. Deductibles are compulsory for all Swiss residents and range from Swiss Francs (CHF) 300 to CHF 2,500 per year. The standard deductible is CHF 300, but insured persons can choose a higher deductible (CHF $500,1,500,2,000$, and 2,500) in exchange for reduced premiums. We performed a retrospective cohort study including all adult persons who were identified as patients with diabetes mellitus, continuously insured, and alive between 2011 (baseline) and 2013 (follow-up). In order to display the temporal sequence of adherence and hospitalization, we determined patients' guideline adherence by including all eligible patients with diabetes between January 1, 2011 and December 31, 2011, and estimated patients' future hospitalization risk on the basis of patients who were still eligible between January 1, 2013 and December 31, 2013. According to the national ethical and legal regulation, an ethical approval was not needed. A written informed patient consent was also not obtained for this study.

\section{Identification of patients with diabetes}

Treated patients were classified as all persons with at least one drug item for diabetes (oral blood glucose-lowering drug [ATC code A10B] or insulin [ATC code A10A]) in the baseline year. According to their drug treatment, we differentiated patients with insulin-dependent from those with noninsulin-dependent diabetes by dividing the sample into three groups: 1) patients using oral antidiabetic agents (OAs), 2) patients using insulin, and 3) patients using OAs and insulin in combination. Additionally, we considered patients' drug treatment with antihypertensives (ATC code C02/C07/ C08/C09) and lipid-lowering agents (ATC code C10) as an indicator for the occurrence of the typical comorbid chronic conditions "hypertension" and "hyperlipidemia".

\section{Definition of adherence to guidelines}

Extracted from the American Diabetes Association recommendations, we identified a set of performance measures reflecting the adherence to guidelines in diabetes care. The set of four performance measures included the frequency of $\mathrm{HbA}_{1 \mathrm{c}}$ measurements, lipid profile (total cholesterol and/or high-density lipoprotein and/or LDL cholesterol and triglycerides), nephropathy status (serum creatinine, albuminuria), and measurement and the frequency of ophthalmologist visits. ${ }^{3}$ Testing the $\mathrm{HbA}_{1 \mathrm{c}}$ was seen as suitable if at least two measurements per patient were performed in 2011, while the other performance measures were defined as adequate if at least one measurement or consultation were conducted in 2011. Good diabetes care in terms of good guideline adherence was seen when all criteria were fulfilled simultaneously. However, to give an insight on how the distribution of the (non)adherent patients looks, the different criteria were broken down into a total of five adherence levels. Thus, a hierarchical model of adherence to guidelines was developed by assigning each performance measure stepwise to an adherence level. The five adherence levels were defined as follows: level 0 was defined as "nonadherent", level 1 included "at least two measurements of $\mathrm{HbA}_{1 \mathrm{c}}$ ", level 2 contained "level 1 and lipid profile", level 3 included "level 2 and nephropathy status", and level 4 was defined as "receiving all performance 


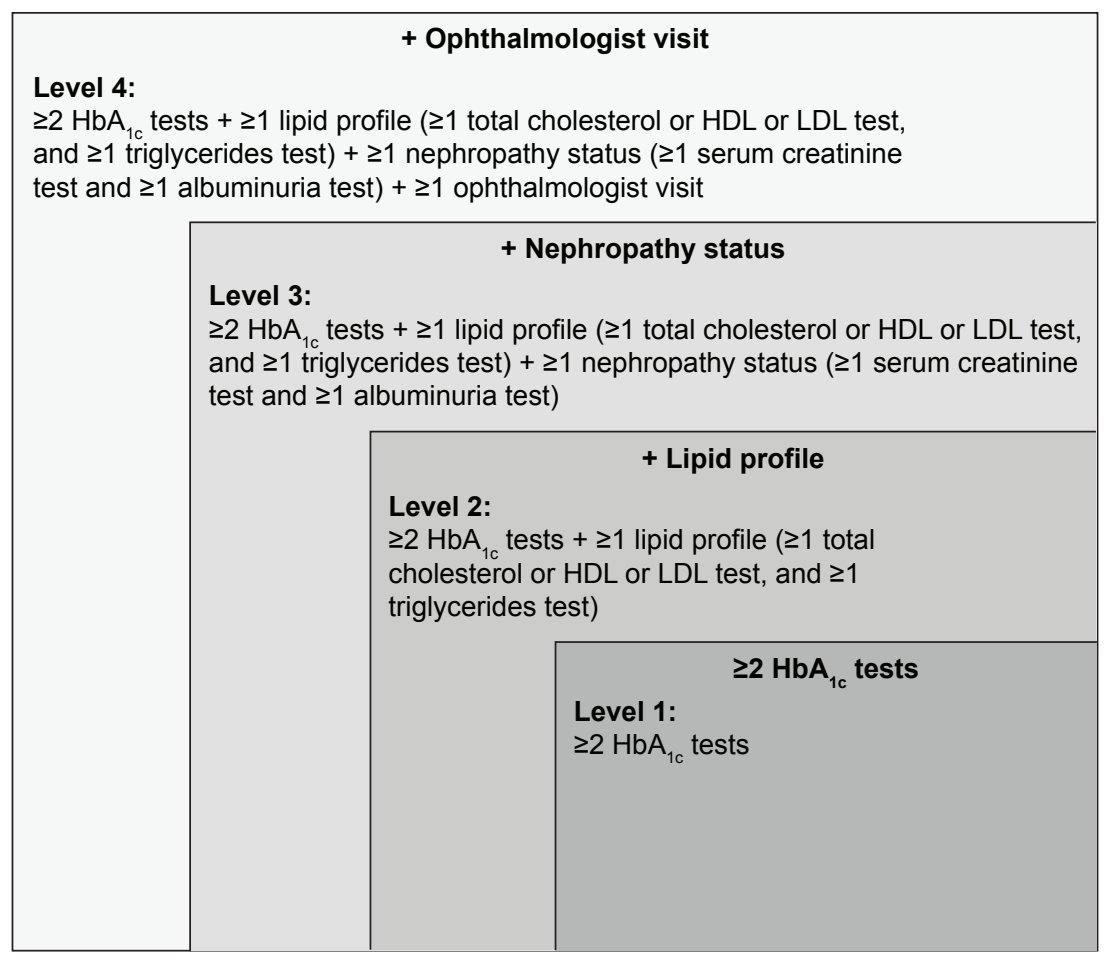

Figure I Classification of adherence levels.

Note: The adherence levels were measured per patient in $20 \mathrm{II}$.

Abbreviations: $\mathrm{HbA}_{\mathrm{Ic}}$, hemoglobin $\mathrm{A}_{\mathrm{Ic}}$; $\mathrm{HDL}$, high-density lipoprotein; LDL, low-density lipoprotein.

measures including ophthalmologist visits" (Figure 1). Thereby a biannual $\mathrm{HbA}_{1 \mathrm{c}}$ measurement was defined as the minimal criterion for guideline adherence. $\mathrm{HbA}_{1 \mathrm{c}}$ testing is the most commonly used measure and preferred standard to monitor glycemic control and represents an indicator to predict complications in patients with diabetes. Consequently, we began with level 0 defined as no or only one $\mathrm{HbA}_{1 \mathrm{c}}$ test per year and no other measurement.

\section{Statistical analysis and outcome}

We used descriptive statistics to characterize the patient group and to examine the performed process measures and the occurrence of hospitalization at baseline. The outcome of interest was all-cause hospitalization in 2013 (follow-up), defined as an overnight hospital stay or a hospital stay of at least 24 hours within the given year. The proportion of patients, who were hospitalized at follow-up, was calculated for each adherence level. We used chi-square tests to compare the differences in the proportions of patients between the three drug treatment groups (OAs, insulin, and OAs and insulin in combination). To predict the probability of hospitalization at follow-up (2013) by the adherence levels of patients with diabetes at baseline (2011), we calculated adjusted odds ratios (OR) and 95\% confidence intervals (CI) using multivariate logistic regression models. Regression analyses were controlled for age, sex, health insurance status, hospitalization in 2011, drug treatment group, drugs used for the treatment of comorbid hypertension and hyperlipidemia, and included an interaction term between adherence level and drug treatment group. The ORs of hospitalization as function of adherence level for the different treatment groups were displayed in a figure. Therefore, we performed two models including the same variables, the first one with adherence in its original scale (as factor) and the second one with adherence treated as interval scaled variable to display the log-linear associations by treatment group. We calculated the effect of adherence levels for the three treatment groups as well as the pairwise differences. $P$-values for all regression model results were calculated with adjustment for multiple comparisons. Data were analyzed using R, version 3.0.1 (R Foundation for Statistical Computing, Vienna, Austria), multiple testing was performed with the supplementary package "multcomp" for multiple testing applying the default comparison procedure. ${ }^{14}$ $P<0.05$ was taken to indicate statistical significance.

\section{Results \\ Patient characteristics}

Of the 40,285 patients included in this cohort, most were males $(55.1 \%)$, over 60 years $(74.8 \%)$, had a low deductible class $(95.4 \%)$, and were not enrolled in a managed care 
model (69.8\%; Table 1). Almost 77\% received at least one prescription for antihypertensive drugs and $52.4 \%$ received a lipid-lowering agent. Overall, 70.5\% were classified as treated with OAs, $12.3 \%$ with insulin, and $17.2 \%$ with OAs and insulin in combination. In every patient group, about three-quarters of patients were aged 60 years and older, with exception of insulin-treated patients who had a significantly higher proportion of those aged under 60 years $(41.1 \%$; $P<0.001)$. The hospitalization rate at follow-up (2013) was lower in patients using OAs than in patients using insulin and in those using OAs and insulin in combination $(26.5 \%$ vs $31.7 \%$ and $31.5 \%$, respectively; $P<0.001$ ).

\section{Performance measures and adherence to guidelines}

The percentages of patients with performed process measures in diabetes care are reported in Table 2 . About $88 \%$ of all patients had at least one annual $\mathrm{HbA}_{1 \mathrm{c}}$ measurement. While $59.0 \%$ of patients had an assessment of the lipid profile, only $19.8 \%$ had undergone a LDL cholesterol test. More than three-quarters of patients had a serum creatinine test, $13.1 \%$ had undergone an albuminuria test, and only $12.0 \%$ had both nephropathy measurements, and $44.2 \%$ visited an ophthalmologist. With exception of the LDL cholesterol test, statistically significant differences between the different drug treatment groups were found for all performance measures. Almost $92 \%$ of patients treated with OAs and insulin in combination had at least one annual $\mathrm{HbA}_{1 \mathrm{c}}$ measurement, whereas only $86.6 \%$ of patients using OAs received this process measure. Furthermore, the proportion of patients visiting an ophthalmologist differs substantially between the patient group "OAs" with $41.2 \%$ and the groups "insulin" and "OAs and insulin in combination" with about $52 \%$.

Approximately $30 \%$ of all patients with diabetes were exposed to nonadherence (level 0 ). In $24.0 \%$ of patients, $\mathrm{HbA}_{1 \mathrm{c}}$ was measured twice a year (level 1). The highest proportion of patients exposed to nonadherence was found at level 2 including at least two $\mathrm{HbA}_{1 \mathrm{c}}$ measurements in a year and an annual lipid profile (37.0\%). About 4\% had in addition to level 2 a yearly nephropathy status (level 3) and only $4.8 \%$ were exposed to the full adherence level (level 4 ), which additionally comprised an annual ophthalmologist visit. Patients treated with OAs were the group with the highest percentage of patients exposed to nonadherence (OAs: $32.9 \%$ vs insulin: $27.0 \%$ and $\mathrm{OAs}$ and insulin in combination: $20.1 \% ; P<0.001)$. Compared with patients treated with OAs and those with OAs and insulin combined, insulin users had the highest proportion of patients exposed to full adherence (level 4; insulin: $7.7 \%$ vs OAs: $3.7 \%$ and OAs and insulin in combination: $7.2 \% ; P<0.001)$.

\section{Adherence and subsequent hospitalization}

Table 3 shows the proportion of patients exposed to (non) adherence in 2011 with a hospitalization in 2013. Almost

Table I Patients characteristics of the diabetes cohort by drug treatment group at baseline

\begin{tabular}{|c|c|c|c|c|c|}
\hline \multirow[t]{2}{*}{ Characteristic } & \multirow{2}{*}{$\begin{array}{l}\begin{array}{l}\text { Total } \\
n=40,285\end{array} \\
\%\end{array}$} & \multirow{2}{*}{$\begin{array}{l}\text { Users of OAs } \\
\mathrm{n}=\mathbf{2 8 , 4 0 4} \\
\%\end{array}$} & \multirow{2}{*}{$\begin{array}{l}\text { Users of insulin } \\
\mathrm{n}=4,965 \\
\%\end{array}$} & \multirow{2}{*}{$\begin{array}{l}\text { Users of OAs and insulin in combination } \\
\mathrm{n}=6,916 \\
\%\end{array}$} & \multirow[t]{2}{*}{$P$-value ${ }^{\text {a }}$} \\
\hline & & & & & \\
\hline \multicolumn{6}{|c|}{ Population characteristics and hospitalization (20 II) } \\
\hline \multicolumn{6}{|l|}{ Sociodemographics } \\
\hline Male & 55.1 & 54.6 & 55.4 & 56.7 & 0.005 \\
\hline Age group (years) & & & & & $<0.001$ \\
\hline $18-39$ & 2.9 & 1.3 & 14.0 & 1.9 & \\
\hline $40-59$ & 22.3 & 21.0 & 27.1 & 23.7 & \\
\hline $60-79$ & 58.2 & 60.4 & 42.5 & 60.6 & \\
\hline$>79$ & 16.6 & 17.3 & 16.4 & 13.8 & \\
\hline \multicolumn{6}{|l|}{ Health insurance status } \\
\hline Managed care model & 30.2 & 31.7 & 25.9 & 27.0 & $<0.001$ \\
\hline Deductible & & & & & $<0.001$ \\
\hline High (>CHF 500) & 4.6 & 5.3 & 2.6 & 3.0 & \\
\hline Low (CHF 300/500) & 95.4 & 94.7 & 97.4 & 97.1 & \\
\hline Hospitalization & 23.4 & 20.5 & 29.4 & 30.9 & $<0.001$ \\
\hline Use of antihypertensive drugs & 76.5 & 77.0 & 63.9 & 83.5 & $<0.001$ \\
\hline Use of lipid-lowering drugs & 52.4 & 51.1 & 45.0 & 62.8 & $<0.001$ \\
\hline Hospitalization (2013) & 25.9 & 26.5 & 31.7 & 31.5 & $<0.001$ \\
\hline
\end{tabular}

Note: ${ }^{a}$-values refer to chi-square test across the three drug treatment groups.

Abbreviations: CHF, Swiss Francs; OAs, oral antidiabetic agents. 
Table 2 Adherence to recommended diabetes care guidelines by drug treatment group

\begin{tabular}{|c|c|c|c|c|c|}
\hline \multirow[t]{2}{*}{ Measure of adherence $(20 \mathrm{II})$} & \multirow{2}{*}{$\begin{array}{l}\text { Total } \\
\%\end{array}$} & \multirow{2}{*}{$\begin{array}{l}\text { Users of } \\
\text { oral OAs } \\
\%\end{array}$} & \multirow{2}{*}{$\begin{array}{l}\text { Users of } \\
\text { insulin } \\
\%\end{array}$} & \multirow{2}{*}{$\begin{array}{l}\text { Users of OAs and } \\
\text { insulin in combination } \\
\%\end{array}$} & \multirow[t]{2}{*}{$P$-value ${ }^{a}$} \\
\hline & & & & & \\
\hline \multicolumn{6}{|l|}{ Performance measures } \\
\hline Annual $\mathrm{HbA}_{\mathrm{Ic}}$ measurement & 87.6 & 86.6 & 87.3 & 91.8 & $<0.001$ \\
\hline \multicolumn{6}{|l|}{ Annual lipid profile } \\
\hline Annual total cholesterol test & 65.2 & 66.6 & 58.3 & 64.2 & $<0.001$ \\
\hline Annual HDL cholesterol test & 56.5 & 57.5 & 52.1 & 55.9 & $<0.001$ \\
\hline Annual LDL cholesterol test & 19.8 & 19.9 & 19.0 & 20.1 & 0.317 \\
\hline Annual triglycerides test & 59.0 & 60.0 & 54.0 & 58.5 & $<0.001$ \\
\hline Annual lipid profile (total cholesterol/HDL/LDL, and triglycerides) & 59.0 & 59.9 & 54.0 & 58.3 & $<0.001$ \\
\hline \multicolumn{6}{|l|}{ Annual nephropathy status } \\
\hline Annual serum creatinine test & 77.4 & 76.7 & 76.6 & 80.6 & $<0.001$ \\
\hline Annual albuminuria test & 13.1 & 11.6 & 17.0 & 16.6 & $<0.001$ \\
\hline Annual nephropathy status (serum creatinine and albuminuria) & 12.0 & 10.6 & 15.5 & 15.4 & $<0.001$ \\
\hline Annual visit to an ophthalmologist & 44.2 & 41.2 & 51.4 & 51.5 & $<0.001$ \\
\hline \multicolumn{6}{|l|}{ Adherence level } \\
\hline Nonadherent (level 0) & 29.9 & 32.9 & 27.0 & 20.1 & $<0.001$ \\
\hline$\geq 2 \mathrm{HbA}_{\mathrm{Ic}}$ measurements in a year (level $\mathrm{I}$ ) & 24.0 & 22.3 & 27.3 & 28.7 & \\
\hline Level I and annual lipid profile (level 2) & 37.0 & 37.1 & 33.7 & 39.1 & \\
\hline Level 2 and nephropathy status (level 3) & 4.3 & 4.1 & 4.3 & 5.0 & \\
\hline Level 3 and visit to an ophthalmologist (level 4) & 4.8 & 3.7 & 7.7 & 7.2 & \\
\hline
\end{tabular}

Note: aP-values refer to chi-square test across the drug treatment groups.

Abbreviations: $\mathrm{HbA}_{1 \mathrm{c}}$, hemoglobin $\mathrm{A}_{\mathrm{Ic}}$; $\mathrm{HDL}$, high-density lipoprotein; LDL, low-density lipoprotein; OAs, oral antidiabetic agents.

one-third of the hospitalized patients were exposed to nonadherence and about $7 \%$ of the patients with hospitalization had at least an adherence level of 3 . Of the patients exposed to nonadherence, $28.2 \%$ were hospitalized, whereas only $19.4 \%$ of the full adherent persons (level 4 ) had a hospital stay (results not shown). The proportion of hospitalized patients using OAs and those using insulin decreased significantly with the adherence levels from over 30\% (OAs: 35.3\%; insulin: $32.2 \%)$ among the patients exposed to nonadherence to $2.8 \%$ (OAs) and $5.7 \%$ (insulin), respectively among the patients exposed to full adherence (level 4; $P<0.001$ ).

The ORs of hospitalization in 2013 as a function of adherence level in 2011 for the different treatment groups, including the interaction term "adherence level-drug treatment group" and adjusted for multiple testing, are shown in Table 4 and Figure 2. The interaction term was statistically significant $(P=0.02$; result not shown). Table 5 shows the results of the model using adherence level as interval scaled variable, which tested the statistical significance of the trends. A significant decreasing trend in the log-odds of hospitalization by adherence level could be observed in all treatment groups (Table $5 ; P<0.001$ ). This linear trend is displayed by thin lines in Figure 2. For the patients treated with insulin, the ORs for hospitalization decreased with each additional level of adherence by a factor of 0.87 (95\% CI: $0.81-0.94)$. Accordingly, the decrease of the ORs was 0.93 (95\% CI:

Table 3 Proportion of patients hospitalized in 2013 by adherence level and drug treatment in $201 \mathrm{I}$

\begin{tabular}{|c|c|c|c|c|c|}
\hline & \multicolumn{5}{|c|}{ Hospitalization (2013) } \\
\hline & Total & $\begin{array}{l}\text { Users } \\
\text { of OAs }\end{array}$ & $\begin{array}{l}\text { Users of } \\
\text { insulin }\end{array}$ & $\begin{array}{l}\text { Users of OAs and insulin } \\
\text { in combination }\end{array}$ & $P_{\text {-value }}{ }^{a}$ \\
\hline & $\%$ & $\%$ & $\%$ & $\%$ & \\
\hline \multicolumn{6}{|l|}{ Adherence level (20I I) } \\
\hline Nonadherent (level 0) & 32.7 & 35.3 & 32.2 & 24.9 & $<0.001$ \\
\hline$\geq 2 \mathrm{HbA}_{\mathrm{Ic}}$ measurement in a year (level I) & 26.9 & 25.0 & 29.6 & 30.9 & \\
\hline Level I and annual lipid profile (level 2) & 33.6 & 33.7 & 29.6 & 36.3 & \\
\hline Level 2 and nephropathy status (level 3) & 3.2 & 3.2 & 2.9 & 3.4 & \\
\hline Level 3 and visit to an ophthalmologist (level 4) & 3.6 & 2.8 & 5.7 & 4.5 & \\
\hline
\end{tabular}

Note: a ${ }^{P}$-values refer to chi-square test across the drug treatment groups.

Abbreviations: $\mathrm{HbA}_{1 \mathrm{c}}$, hemoglobin $\mathrm{A}_{\mathrm{lc}}$; OAs, oral antidiabetic agents. 
Table 4 Prediction of hospitalization by adherence level (as factor variable), including the interaction term "adherence level-drug treatment group" and adjusting for multiple comparisons

\begin{tabular}{|c|c|c|c|c|c|c|}
\hline & \multicolumn{6}{|c|}{ Hospitalization $(2013)$} \\
\hline & \multicolumn{2}{|l|}{ Users of insulin } & \multicolumn{2}{|c|}{ Users of oral OAs } & \multicolumn{2}{|c|}{$\begin{array}{l}\text { Users of OAs } \\
\text { and insulin in } \\
\text { combination }\end{array}$} \\
\hline & OR $^{\mathbf{a}}$ & $95 \% \mathrm{Cl}$ & OR $^{\mathbf{a}}$ & $95 \% \mathrm{Cl}$ & OR $^{\mathbf{a}}$ & $95 \% \mathrm{Cl}$ \\
\hline \multicolumn{7}{|l|}{ Adherence level (20II) } \\
\hline Nonadherent (level 0) & 1.00 (reference) & & 0.55 & $0.45-0.66$ & 0.94 & $0.74-1.20$ \\
\hline$\geq 2 \mathrm{HbA}_{\mathrm{Ic}}$ measurement in a year (level I) & 0.88 & $0.69-1.13$ & $0.55^{\mathrm{d}}$ & $0.45-0.67$ & $0.78^{c}$ & $0.62-0.97$ \\
\hline Level I and annual lipid profile (level 2) & $0.74^{b}$ & $0.58-0.94$ & $0.48^{\mathrm{d}}$ & $0.40-0.58$ & $0.70^{d}$ & $0.57-0.87$ \\
\hline Level 2 and nephropathy status (level 3 ) & $0.57^{c}$ & $0.34-0.96$ & $0.47^{d}$ & $0.35-0.62$ & $0.54^{d}$ & $0.36-0.83$ \\
\hline Level 3 and visit to an ophthalmologist (level 4) & $0.64^{\mathrm{c}}$ & $0.43-0.95$ & $0.39^{d}$ & $0.29-0.53$ & $0.43^{d}$ & $0.30-0.63$ \\
\hline
\end{tabular}

Notes: ${ }^{a}$ Adjusted for sociodemographics, health insurance status, hospitalization, use of antihypertensive drugs, and use of lipid lowering drugs; ${ }^{b} P \leq 0.0 \mathrm{I} ;{ }^{c} P \leq 0.05$; ${ }^{d} P \leq 0.00 \mathrm{I}$. Abbreviations: $\mathrm{Cl}$, confidence interval; $\mathrm{HbA}_{1 \mathrm{c}}$, hemoglobin $\mathrm{A}_{\mathrm{lc}}$; OAs, oral antidiabetic agents; OR, odds ratio.

$0.90-0.97)$ among patients treated with OAs and $0.84(95 \%$ CI: 0.79-0.90) among those treated with OAs and insulin in combination. When comparing the trends between the treatment groups, the effect of adherence on the hospitalization risk was strongest in patients treated with OAs and insulin in combination. However, the effect in this treatment group is only significantly greater than the effect of the treatment group with OAs only $(P=0.002)$. The other two differences in trends were not significantly different.

\section{Discussion}

Our study revealed four findings. First of all, physicians' guideline adherence, reflected by a simple set of performance measures was quite low. Second, adherence increased with a more intensified therapy and severity of diabetes. Third, guideline adherence predicted the risk of hospitalization. And finally, the effect size of the association between

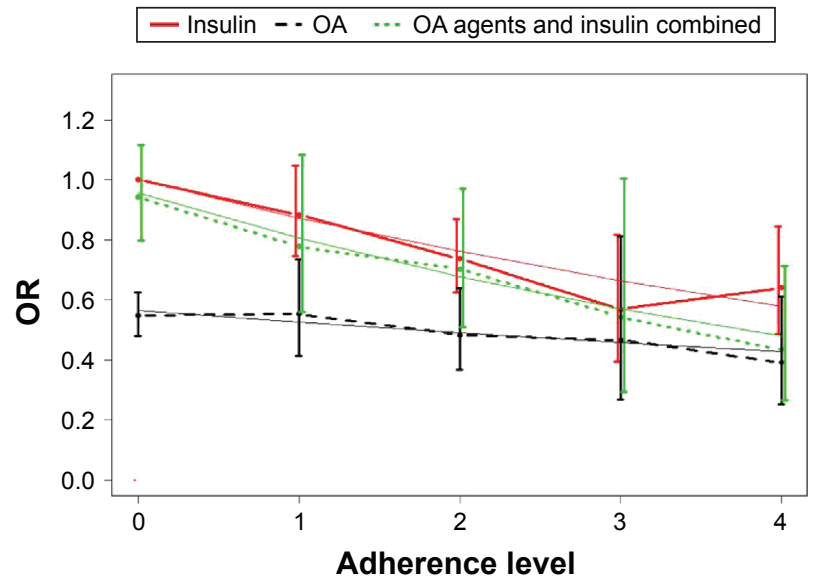

Figure 2 Odds ratios of hospitalization as a function of adherence level by treatment group.

Note: The thin lines represent the linear trend.

Abbreviations: OAs, oral antidiabetic agents; OR, odds ratio. adherence and hospitalization risk depended on the therapy intensity.

Surprisingly, only a small percentage of patients $(5 \%)$ were exposed to full adherence including the performance of all recommended process measures. About $30 \%$ of patients were considered as exposed to nonadherence and did not receive at least a biannual $\mathrm{HbA}_{1 \mathrm{c}}$ measurement. These results are consistent with prior research, which highlighted the poor guideline adherence in diabetes care, especially in the case of the $\mathrm{HbA}_{1 \mathrm{c}}$ measurement as the most important measure in the diabetes management. ${ }^{15,16}$ Results from an US study showed that only $70 \%$ of patients with diabetes received an annual $\mathrm{HbA}_{1 \mathrm{c}}$ measurement and a large cohort study from Luxembourg reported that even $55 \%$ of patients had no $\mathrm{HbA}_{1 \mathrm{c}}$ test within a year. ${ }^{15,16}$ Furthermore, our study revealed a poor adherence with $20 \%$ in the measurement of LDL cholesterol, which is classified as an essential parameter in testing lipid profile in national

Table 5 Prediction of hospitalization by adherence level (as interval scaled variable), including the interaction term "adherence level-drug treatment group" and adjusting for multiple comparisons

\begin{tabular}{llc}
\hline & \multicolumn{2}{l}{$\begin{array}{l}\text { Hospitalization (20 I3) by } \\
\text { Adherence level (20I I) }\end{array}$} \\
\cline { 2 - 3 } & OR $^{\text {a }}$ & $\mathbf{9 5 \% ~ C l ~}$ \\
\hline Drug treatment group & & \\
Insulin & $0.87^{\mathrm{b}}$ & $0.8 \mathrm{I}-0.94$ \\
OAs & $0.93^{\mathrm{b}}$ & $0.90-0.97$ \\
OAs and insulin in combination & $0.84^{\mathrm{b}}$ & $0.79-0.90$ \\
OAs vs insulin & 1.07 & $0.98-1.16$ \\
OAs and insulin in combination vs insulin & 0.96 & $0.87-1.06$ \\
OAs and insulin in combination vs OAs & $0.90^{\mathrm{c}}$ & $0.84-0.97$ \\
\hline
\end{tabular}

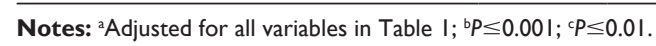

Abbreviations: $\mathrm{Cl}$, confidence interval; OAs, oral antidiabetic agents; OR, odds ratio. 
as well as in international recommendations in diabetes care. ${ }^{4,17}$ Previous studies from Switzerland also suggested a suboptimal performance of $\mathrm{HbA}_{1 \mathrm{c}}$ measurement and lipid profile assessment, but showed higher adherence levels in lipid profile assessment than in our study. Bovier et al, ${ }^{18}$ reported $\mathrm{HbA}_{1 \mathrm{c}}$ measurements three times a year for $65 \%$ and a yearly lipid profile assessment for $89 \%$ in a diabetes sample of 400 patients in the French-speaking part of Switzerland. A further study showed that $84 \%$ had a biannual $\mathrm{HbA}_{1 \mathrm{c}}$ measurement among $\mathrm{HbA}_{1 \mathrm{c}}$ aware patients and $94 \%$ had undergone a lipid profile assessment. ${ }^{19}$ However, comparability to our findings is limited since previous studies were based on patients' self-reporting, older data, or on selected medical files of a small diabetes sample and not on a large general population of persons with diabetes. Most patients achieved only adherence level 2 including at least two $\mathrm{HbA}_{1 \mathrm{c}}$ measurements and a lipid profile assessment. The proportion of patients with an additional nephropathy status (level 3) and ophthalmologist consultation (level 4) decreased significantly.

Therapy intensity, reflected in an additional insulin therapy or insulin therapy only was associated with an increased guideline adherence: we found the highest proportion of patients receiving care according to level 4 (full adherence) in insulin users, followed by those with combined therapy (OA agents and insulin). We suppose that with increased disease severity, reflected in an intensified therapy, physicians' awareness and therefore guideline adherence increased.

The major finding of our study is that it provides evidence that the adherence to medical standards in diabetes care led to a significantly decreased probability of future hospitalization among patients. An interaction between adherence levels and drug treatment group was found showing different trends for the treatment groups. The hospitalization risk for the treatment group with OAs was for low adherence levels considerably smaller than for the other two treatment groups. The effect size of the association between adherence and hospitalization risk was significantly stronger in the OAs group compared with the treatment group with OAs and insulin in combination. The association between adherence to guidelines and reduced rates of hospitalization was also found in the study by Sloan et al. ${ }^{11}$ Only few comparable population-based studies examining the impact of adherence to medical standards on outcomes such as hospitalization are available, particularly those using performed process measures as indicator for the adherence to guidelines. Most studies predicting health outcomes such as hospitalization or mortality are focused on oral antihyperglycemic medication adherence. ${ }^{20-23}$

In this context, it is important to acknowledge that guideline adherence in our study was defined by simple performance measures. Our results suggest that even this simple measurement, which could be easily calculated from health care claims data, is able to predict outcomes such as hospitalization. In Switzerland - as in many countries - there is a huge discussion on how to measure quality of care in ambulatory care. Our results suggest that already available datasets could help to reflect quality of care and therefore help to improve it.

Several strengths and limitations of our study have to be taken into account. The main strength is that the study was based on very comprehensive health care claims data, which covers a large population-based cohort of patients with diabetes. Health care claims data are a reliable and practicebased information source, and they are particularly valuable when data from population-based disease studies are lacking. Our findings confirm that claims-based adherence measures are good indicators to determine patients' health care behavior and are additionally valuable predictors for health care utilization. The study also has several limitations. First, the number of patients with diabetes may be biased, because clinical diagnoses (eg, ICD-10) were not available, and undiagnosed patients without medication but with lifestyle treatment could not be included in the study. However, we assume that this potential bias is negligible, since diagnoses based on dispensed drugs are a valid proxy for clinical diagnoses and in the meanwhile widely used in epidemiological and outcomes research to estimate diabetes prevalence. ${ }^{24,25}$ Second, since we used the World Health Organization ATC codes to identify patients with diabetes, we were unable to distinguish between type 1 and type 2 diabetes. However, according to epidemiological data, it is assumed that $~ 90 \%$ of patients with diabetes were classified with type 2 diabetes. Moreover, since patients with type 1 diabetes are mostly younger persons ( $\leq 18$ years), and we included only adult patients, the majority of our study sample most likely comprised patients with type 2 diabetes. ${ }^{26}$ Third, the used data did not allow us to investigate long-term effects of poor adherence on health outcomes, thus it would be clinically important to examine a longer observation period in future studies. Fourth, our data did not include further information on clinical parameters such as laboratory values, duration of diabetes, and cardiovascular risk factors (ie, blood pressure and body weight). Although our analysis was adjusted for numerous variables indicating patients' disease severity 
(kind of drug treatment) or comorbidities (eg, using drugs for hypertension), our information on the severity of diabetes and the overall health status is limited. A poor health status can act as confounder in our analyses. Since the frequency of health care use strongly depends on the disease severity, an underestimation of the positive impact on guideline adherence cannot be excluded. Fifth, the analyses criteria were process indicators and not intermediate ones. Guideline adherence has therefore to be considered as a proxy for better care in terms of better communication, education, or methodological approach. Finally, according to the given cohort study design, we had to exclude dead or ineligible persons from the study population. Therefore, the impact of guideline adherence and hospitalization risk applies only to persons who survive long enough to measure the adherence and its potential effect on future outcomes.

\section{Conclusion}

This study showed that health care claims data can be used to calculate a simple set of performance measures to observe adherence of provided care to established guideline recommendations. Furthermore, a clear relation between adherence levels and the hospitalization probability of diabetes patients could be proven. Regarding the low performance in the observed measures, our study emphasizes the need to foster the implementation of evidence in daily treatment of diabetes patients. Simple measures provided in this study seem to be able to enable this.

\section{Acknowledgments}

This study received funding from Merck, Sharp and Dohme (MSD), Switzerland. We thank MSD for supporting our study. CAH, RR, and OR received funding from MSD. MB received consulting fees from MSD. TR received no funding. The sponsor had no role in collection, analysis, and interpretation of data; writing of the paper; or in the decision to submit the paper for publication.

\section{Author contributions}

All authors contributed substantially to conceptual development, the study design, and interpretation of data. CAH and RR analyzed the data and CAH drafted the manuscript. All authors critically revised the article for important intellectual contents and gave the final approval of the version to be published. Furthermore, they agreed to be accountable for all aspects of the work in ensuring that questions related to the accuracy or integrity of any part of the work are appropriately investigated and resolved.

\section{Disclosure}

The authors report no conflicts of interest in this work.

\section{References}

1. Tamayo T, Rosenbauer J, Wild SH, et al. Diabetes in Europe: an update. Diabetes Res Clin Pract. 2014;103:206-217.

2. Danaei G, Finucane MM, Lu Y, et al. National, regional, and global trends in fasting plasma glucose and diabetes prevalence since 1980: systematic analysis of health examination surveys and epidemiological studies with 370 country-years and 2.7 million participants. Lancet. 2011; 378:31-40.

3. American Diabetes Association. Standards of Medical Care in Diabetes - 2013. Diabetes Care. 2013;36:S11-S66.

4. Rydén L, Grant PJ, Anker SD, et al. ESC Guidelines on diabetes, prediabetes, and cardiovascular diseases developed in collaboration with the EASD: the Task Force on diabetes, pre-diabetes, and cardiovascular diseases of the European Society of Cardiology (ESC) and developed in collaboration with the European Association for the Study of Diabetes (EASD). Eur Heart J. 2013;34:3035-3087.

5. Ahmann AJ. Guidelines and performance measures for diabetes. Am J Manag Care. 2007;13(Suppl 2):S41-S46.

6. De Berardis G, Pellegrini F, Franciosi M, et al. Quality of care and outcomes in type 2 diabetic patients: a comparison between general practice and diabetes clinics. Diabetes Care. 2004;27:398-406.

7. Bell RA, Camacho F, Goonan K, et al. Quality of diabetes care among lowincome patients in North Carolina. Am J Prev Med. 2001;21:124-131.

8. Fleming BB, Greenfield S, Engelgau MM, Pogach LM, Clauser SB, Parrott MA. The Diabetes Quality Improvement Project: moving science into health policy to gain an edge on the diabetes epidemic. Diabetes Care. 2001;24:1815-1820.

9. Giorda C, Picariello R, Nada E, et al. The impact of adherence to screening guidelines and of diabetes clinics referral on morbidity and mortality in diabetes. PLoS One. 2012; 7:e33839.

10. Oh SW, Lee HJ, Chin HJ, Hwang JI. Adherence to clinical practice guidelines and outcomes in diabetic patients. Int J Qual Health Care. 2011; 23:413-419.

11. Sloan FA, Bethel MA, Lee PP, Brown DS, Feinglos MN. Adherence to guidelines and its effects on hospitalizations with complications of type 2 diabetes. Rev Diabet Stud. 2004;1:29-38.

12. Giorda CB, Picariello R, Nada E, et al. Comparison of direct costs of type 2 diabetes care: different care models with different outcomes. Nutr Metab Cardiovasc Dis. 2014;24:717-724.

13. WHO Collaborating Centre for Drug Statistics Methodology. Guidelines for ATC classification and DDD assignment 2011. Oslo; 2010.

14. Hothorn T, Bretz F, Westfall P. Simultaneous inference in general parametric models. Biom J. 2008;50:346-363.

15. Beckles GL, Engelgau MM, Narayan KM, Herman WH, Aubert RE, Williamson DF. Population-based assessment of the level of care among adults with diabetes in the U.S. Diabetes Care. 1998;21:1432-1438.

16. Renard LM, Bocquet V, Vidal-Trecan G, Lair ML, Blum-Boisgard C. Adherence to international follow-up guidelines in type 2 diabetes: a longitudinal cohort study in Luxembourg. PLoS One. 2013;8: e80162.

17. Swiss Society for Endocrinology and Diabetology. Criteria for a "good" disease management. Available from: http://www.sgedssed.ch/fileadmin/ files/6_empfehlungen_fachpersonen/63_praxis-empfehlungen/ Kriterien-DMD_v2014_14-08-19.pdf. Accessed February 11, 2016.

18. Bovier PA, Sebo P, Abetel G, George F, Stalder H. Adherence to recommended standards of diabetes care by Swiss primary care physicians. Swiss Med Wkly. 2007;137:173-181.

19. Zuercher E, Casillas A, Hagon-Traub I, Bordet J, Burnand B, PeytremannBridevaux I. Baseline data of a population-based cohort of patients with diabetes in Switzerland (CoDiab-VD). Swiss Med Wkly. 2014; 144:w13951.

20. Krass I, Schieback P, Dhippayom T. Adherence to diabetes medication: a systematic review. Diabet Med. 2015;32:725-737. 
21. Lau DT, Nau DP. Oral antihyperglycemic medication nonadherence and subsequent hospitalization among individuals with type 2 diabetes Diabetes Care. 2004;27:2149-2153.

22. Sokol MC, McGuigan KA, Verbrugge RR, Epstein RS. Impact of medication adherence on hospitalization risk and healthcare cost. Med Care. 2005;43:521-530.

23. Ho PM, Rumsfeld JS, Masoudi FA, et al. Effect of medication nonadherence on hospitalization and mortality among patients with diabetes mellitus. Arch Intern Med. 2006;166:1836-1841.

24. Tu K, Manuel D, Lam K, Kavanagh D, Mitiku TF, Guo H. Diabetics can be identified in an electronic medical record using laboratory tests and prescriptions. J Clin Epidemiol. 2011;64:431-435.
25. O'Shea M, Teeling M, Bennett K. The prevalence and ingredient cost of chronic comorbidity in the Irish elderly population with medication treated type 2 diabetes: a retrospective cross-sectional study using a national pharmacy claims database. BMC Health Serv Res. 2013;13:23.

26. International Diabetes Federation. IDF Diabetes Atlas. 6th ed. Brussels: International Diabetes Federation; 2013.

\section{Publish your work in this journal}

Patient Preference and Adherence is an international, peer-reviewed, open access journal that focuses on the growing importance of patient preference and adherence throughout the therapeutic continuum. Patien satisfaction, acceptability, quality of life, compliance, persistence and their role in developing new therapeutic modalities and compounds to optimize clinical outcomes for existing disease states are major areas of interest for the journal. This journal has been accepted for indexing on PubMed Central. The manuscript management system is completely online and includes a very quick and fair peer-review system, which is all easy to use. Visit http://www. dovepress.com/testimonials.php to read real quotes from published authors.

Submit your manuscript here: http://www.dovepress.com/patient-preference-and-adherence-journal 